\title{
Low Latency 5G Distributed Wireless Network Architecture: A Techno-Economic Comparison
}

\author{
Ibrahim Alhassan Gedel * and Nnamdi I. Nwulu * \\ Department of Electrical and Electronic Engineering Science, Corner Kingsway and University Johannesburg, \\ Johannesburg 2092, South Africa \\ * Correspondence: gedel.ibrahim@gmail.com (I.A.G.); nnwulu@uj.ac.za (N.I.N.)
}

\section{check for} updates

Citation: Gedel, I.A.; Nwulu, N.I. Low Latency 5G Distributed Wireless Network Architecture: A

Techno-Economic Comparison. Inventions 2021, 6, 11. https://doi. org/10.3390/inventions6010011

Received: 11 October 2020

Accepted: 1 December 2020

Published: 24 January 2021

Publisher's Note: MDPI stays neutral with regard to jurisdictional claims in published maps and institutional affiliations.

Copyright: (c) 2021 by the authors. Licensee MDPI, Basel, Switzerland. This article is an open access article distributed under the terms and conditions of the Creative Commons Attribution (CC BY) license (https:// creativecommons.org/licenses/by/ $4.0 /)$.

\begin{abstract}
The most profound requirements of fifth-generation (5G) technology implementations are the architecture design and the radio base station technology to capably run applications such as device-to-device, machine-to machine and internet of things at a reduced latency. Owing to these requirements, the implementation of $5 \mathrm{G}$ technology is very expensive to mobile network operators (MNO). In this study we modified the existing $4 \mathrm{G}$ network to form a distributed wireless network architecture (DWNA); the picocell and distributed antenna system were modified to support the enabling technology of 5G technology were a multi-edge computer (MEC), software-defined networking (SDN), massive multiple input multiple output (MIMO), ultra-dense network (UDN), Network Functions Virtualization (NFV) and device-to-device (D2D) communication at a reduced cost of ownership, improved coverage and capacity. We present a mathematical model for operational expenditure, capital expenditure and total cost of ownership (TCO) for the DWNA. A mathematical model for DWNA capacity and throughput was presented. Result shows that it is very economical for MNO to rent the space of the tower infrastructure from tower companies. The sensitivity analysis also shows a significant reduction in TCO for both the modified picocell and modified distributed antenna systems.
\end{abstract}

Keywords: 5G technology; capital expenditure; operational expenditure; total cost of ownership; distributed wireless network architectures

\section{Introduction}

The introduction of new services such as device-to-device (D2D) communication, machine-to-machine (M2M) communication and the internet of things (IoT), as well as the increased demand for mobile data, have necessitated the implementation of fifth-generation (5G) technology [1]. This is due to the dense coverage, high capacity, low latency and high quality of service that can be achieved with 5G technology. The 5G will require gNodeB (macrocell) with other technology such as microcell, radio remote head (RRH), distributed antenna system (DAS), relay-based systems, picocell and femtocell to form a heterogeneous network. Cognitive radio, network function virtualization, software-defined networking (SDN), ultra-density and multiple input multiple output (MIMO) are other key 5G technologies. MIMO and massive MIMO technology will be integrated with 5G to improve network coverage, capacity and data rate at the macrocell level further. The $5 \mathrm{G}$ will further enhance the coverage, capacity, energy efficiency and spectral efficiency in a dead signal location.

Ultra-broadband backhaul links can be achieve using millimetre wave (mmWave) technology, this is to carry traffic to or from either small base stations (BSs) or relay stations in 5G. The 5G technology can be either centralized or distributed architecture, depending on how the baseband units and switch are deigned. The current $2 \mathrm{G}, 3 \mathrm{G}$ and $4 \mathrm{G}$ networks are all distributed, with each cell having it baseband unit interconnected from the end site to the backbone. The major backbone to the base station controller (BSC), universal mobile telecommunications system radio access network (URAN) and the evolved packet core 
(EPC). These networks are complex, high-latency, high intercell interference and required serval hand-off during movement. The technology is not capable of handling the current data requirement and modern applications such as D2D, IoT and M2M. Although a lot of study on $5 \mathrm{G}$ shows that there will be a complete change in the radio access network and the core network, this study shows otherwise. This study is necessary to reduce the capital expenditure (Capex) and operational expenditure (Opex), but at the same time meet all the requirements of $5 \mathrm{G}$ technology to reduce low latency. The study proposed a distributed wireless network architecture (DWNA) to complement the drawbacks of the centralized wireless network architecture (CWNA) for 5G deployment. Unlike the CWNA, the DWNA will reduce Capex and Opex, improve network security, reduce traffic bottlenecks and reduce the cost of transmission backhaul and fronthaul, since the architecture will have a cluster of baseband units (BBU) and small centralized radio access network (C-RAN), which will again reduce latency because the subscribers are closed the C-RAN and the switching is better than that of the CWNA. DWNA is used in bridging a number of access point, for examples buildings or offices, through a wireless local area network [2].

\section{Structure of the Paper}

The paper is organised as follows: in Section 3, we do a review of a related works in the area of study. Section 4, we give detail description of the proposed architecture for the $5 \mathrm{G}$ mobile technology. Section 5 is the techno-economic model formulation for the distributed wireless network, Section 6 is the network capacity and throughput model formulation. Section 7 we do the model verification. In Section 8 is the model simulation and in Section 9 is the conclusion followed by the referencing.

\section{Review of Related Works}

The level of spatial densification in a 5G wireless network necessitates the use of small cells to form a distributed wireless network where mmWave technology is used for communication or data transfer $[3,4]$ between cells and a multiprotocol label switching (MPLS) network or a fiber network is used for data transfer to the core network.

Many scholars have recommended the use of mmWave for small cell $5 \mathrm{G}$ heterogamous networks. mmWave technology is more dependable when it comes to attenuation due to rain, provided the required spectrum is used with small cell technology, especially for indoor use. mmWave is more efficient when used with directional antennas.

The proposed architecture will run on internet protocol (IP) backhauling and fronthauling using fiber-optic technology. mmWave will also be used as fronthauling from the RRH to the BBU for traffic offloading. The mmWave technology will change backhauling techniques in the mobile industry owing to the available spectrum at this band. The mmWave will help to reduce antenna sizes, enabling the fabrication of hundreds or thousands of antenna elements, even at the user equipment (UE). The start of 5G happens together with a global economic crisis. Mobile network operators (MNO) can never terminate investment in innovative technologies to obtain profits without any economic analysis.

According to reference [5], MNO will require augmentation of a 5G new ratio (NR) or gNodeB to be able to render modern services such as IoT, D2D and M2M communication. This will require better and cost-effective planning. Reference [6] presented an analysis comparing Capex, Opex, total cost of ownership (TCO), and network capacity based on antenna expansion, intercell interference and energy efficiency for a distributed antenna system (DAS) and femtocell. Reference [2] examined the techno-economic analysis of various small cell and DAS financial models, Capex and Opex.

Reference [1] defined TCO for DAS as the sum of Capex and Opex and a sensitivity analysis was done on the bandwidth, running cost and interest rate. Reference [7] presented a techno-economic analysis for 5G architecture for DAS and MIMO. Reference [8] used an ordinary annuity model to predict the future annual repeating payment of the principal amount invested in Capex, Opex and TCO. Reference [9] stated that the principal cost for operations includes the running cost and backhaul cost, whereas the principal invested 
capital amount is made up of the eNodeB and the EPC, as well as the transmit and receive antenna.

Reference stated that [10] infrastructure sharing will enhance the deployment of 5G technologies and will help MNOs meet the dense coverage, high capacity and reduced latency requirements. Passive infrastructure sharing will spread the infrastructure expenditure of 5G implementation among multiple players, which will reduce cost and increase profitability.

Reference [11] argued that telecommunication infrastructure sharing will allow independent tower companies to manage the infrastructure, while the MNO focus on providing the best telecom service with no Capex and very limited Opex. A study by [12], shows that 5G infrastructure cost in Britain is very high and the economical way to reduce the cost of infrastructure is by infrastructure sharing and network virtualization [13]. This paper will develop a mathematical model that will be used to demonstrate how infrastructure sharing can be used to reduce the total cost of ownership of 5G network implementation.

In reference [14] illustrate that the reconstruction of a new architecture will improve level of performance, enhance energy efficiency, and decreases Capex, Opex and TC. The author of the paper [15] stated that the key enabling technologies of 5G technology are multi-edge computer (MEC), SDN, massive MIMO, ultra-dense network (UDN), NFV and D2D communication. In reference [14], reviewed several network architectures this includes partial C-RAN which has L1 functions in the RRH and both L2 and L3 are in BBU and full C-RAN is when we have LI, L2, L3 functionally are placed in the BBU. A study by [14] revealed that in a full C-RAN, resources are in a pool and are dynamically shared to reduce transmission congestion, reduced latency, improve performance, improve throughput, then reduce power consumption and increase network flexibility [16]. Another author also proposed a new RAN architecture named the heterogamous CRAN or H-CRAN, which has both the user plan and control plan in decoupled.

Although much has been written on the techno-economic model and sensitivity analysis of some essential variables in literature, researchers have not considered the assessment of 5G DWNA and the techno-economic model for distributed network architecture in 5G. References $[2,6,8,10,17]$ present technoeconomic models and sensitivity analysis for DAS, MIMO, macrocells and small cells, but the models presented do not take into account the effects of inflation, the interest rate, and some key variables on investments in 5G, coupled with DWNA. From the aforementioned literature, no study has concurrently assessed the effect of interest rate and inflation on the proposed economic model for 5G DWNA. The key contributions of this study are highlighted below:

Proposed 5G long-term evolution (LTE) DWNAs designed with modified picocell (MPICOC), modified DAS (MDAS; small cell) and femtocell.

A mathematical model for Capex, Opex and TCO will be presented for 5G distributed MPICOC, MDAS (small cell) and femtocell and the model for DWNAs.

This study will investigate the Capex, Opex and TCO for the MPicocell and MDAS when the MNO owns the tower infrastructure and when the tower infrastructure belongs to tower companies.

A mathematical model of network capacity and throughput for a distributed wireless network using the Shannon capacity theory will be presented.

A sensitivity analysis (SA) will be performed to evaluate the Capex, Opex and TCO with MIMO on MPICOC and MDAS. This will be repeated for TCO with MIMO on MPICOC and MDAS

\section{Fifth-Generation (5G) Long-Term Evolution (LTE)—Distributed Wireless Network Architectures}

Figure 1 below shows a proposed DWNA for a 5G LTE, using architecture comprising MPICOC for improving the RRH, MIMO and BBU to meet the 5G technology requirement at a reduced TCO. The MPICOC encompasses centralized BBU, RRH and MIMO connected to a localised data centre called a MEC in the cloud through fiber optics whiles the MPICOCs are distributed connected hence the proposed architecture, the existing picocell is modified 
to support different applications, to support key enabling technology of 5G technology are MEC, SDN, massive MIMO, ultra-dense network (UDN), NFV and D2D communication. The BBU is centralized with L1, L2, L3 functionally, both the MEC and BBU run on a SDN. The implementation SDN will help in improving bandwidth, reliability, availability, monitoring, and end-to-end management capabilities. SDN will separate all the control interface from the individual device, and then create a dynamic, manageable, cost-effective, and adaptable architecture that gives administrators unprecedented automation, and control resulting in reduction in latency. SDN implementation will improve resources utilization, fault finding, and makes resolution very easy. Again, the use of SDN will optimize the performance of a mobile backhaul network by dynamically finding the best backhaul route assigning required wavelength, and the location of the local base band unit.

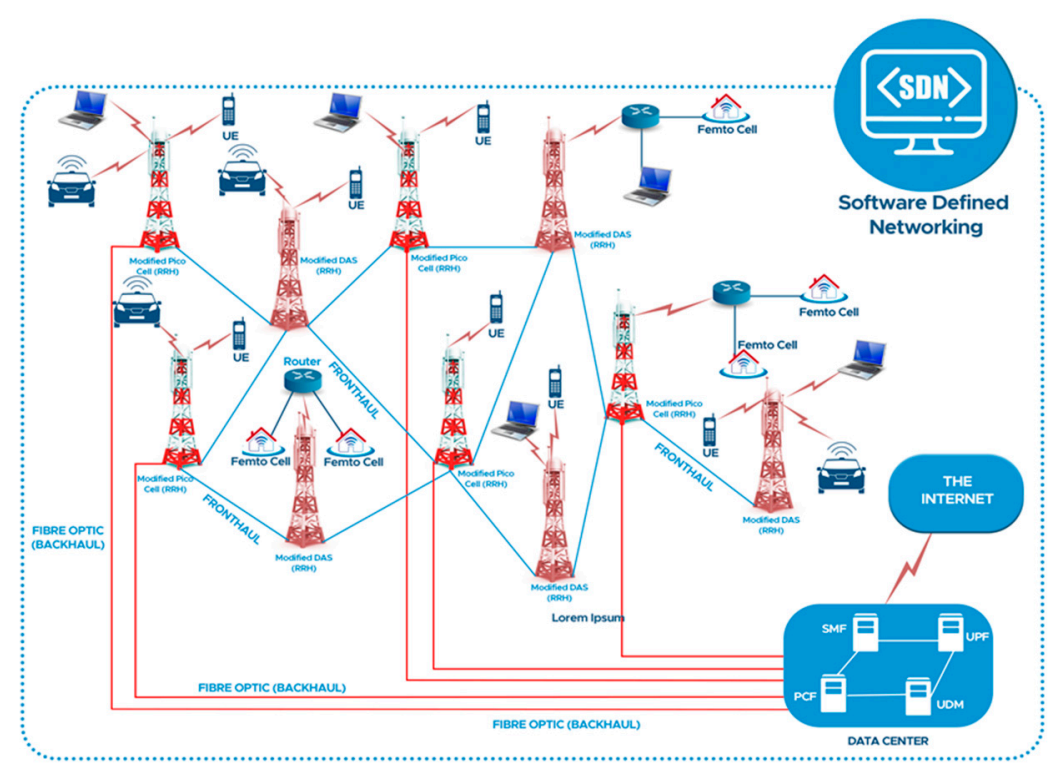

Figure 1. Fifth-generation (5G) wireless backhauling network for a distributed system with centralized radio access network (C-RAN).

The MDAS is modified to offload traffic to the MPICOC through a fronthaul network. The MPICOCs are centralized connected to over 50 to 100 MDAS in a cluster. The MDAS is made up of RRH with MIMO technology, improved bandwidth, support IP, improved process speed by moving L1 and L2 overheads to the BBU and to support the new frequency band of $5 \mathrm{G}$ technology. The RRH are linked to the BBU through a fronthaul network. The proposed MPICOC and MDAS architecture will drastically reduce latency, since there is no handing over between the MDAS and MPICOC; moreover, dense coverage and high capacity are achieved owing to the number of MDASs and the distance between MDASs. The architecture will use the femtocell as an indoor device and traffic will be offloaded through mmWave, which is the same for a broadband device. The proposed architecture is also integrated with SDN located at a central point and controlling all the operations and maintenance on the MDAS, MPICOC, the fronthaul network and the IP network. The backhaul network is an IP network connecting all the MPICOC to the multi-edge computer in the cloud. Edge computing architecture is C-RAN technology designed to reduce latency. This is achieved by locating the key processing task closer to the user to allow all the data gathered to be processed very close to the end user instead of transmitting it to a centralized server. The edge computing techniques proposed are implemented on IoT and D2D technology to reduce latency. The NR or the gNodeB are special BSs designed to run several services, which include IoT, D2D and M2M communication, all through the mmWave spectrum. The MDAS will reduce energy consumption by $75 \%$, since no cooling is needed, nor BBU, and maintenance occurs bi-annually, making this architecture most 
economical. The architecture makes it quite easy to extend coverage and increase capacity at a very reduced cost.

\section{Techno-Economic Model Formulation}

In this section, we built on [6] to improve the economic model for an annual repeating payment for the telecom infrastructure and the TCO or cost of investment.

$$
\begin{gathered}
\varepsilon_{i}=\sum_{k=0}^{K-1} \frac{\alpha_{k, i}}{(1+\beta)^{k}} \\
A=P \frac{r(1+r)^{n}}{(1+r)^{n}-1} \\
A=\frac{i}{1-(1+i)^{-n}} C \\
C_{\text {macro }}=\frac{i}{1-(1+i)^{-n}} N\left(C_{e N B}+C_{E P C}\right)+f_{\text {site }} \frac{i}{1-(1+i)^{-n}} N\left(C_{e N B}+C_{E P C}\right)+N_{C_{\text {site }}}+f_{B W} B W \\
C_{\text {macro }}^{T C O}=\left(1+f_{m}\right) N\left(C_{e N B}+C_{E P C}\right) \frac{r(1+r)^{n}}{(1+r)^{n-1}}+N C_{s t}+f_{B W} B W \\
C_{\text {small }}=\left(1+f_{\text {site }}\right) \frac{i}{1-(1+i)^{-n}} N C_{\frac{i}{f}}
\end{gathered}
$$

Equation (1) is a repeating payment model where $\varepsilon_{i}$ is the recurring payment, $\alpha_{i}$ denotes the present value of the cost of individual, $\beta$ symbolizes the interest rate and $k$ denotes the years of repayment. The parameter $n$ in all the equations above designates the annual repayment plan. Again, Equations (2) and (3) [17] are the same as $1, C=P=\varepsilon_{i}$, $r=I=\beta$ and $n=n=k$. Equation (4) $[7,8]$ is the TCO for a macrocell with parameters including $N$ is the number of eNodeB, $C_{e N B}$ is the capital cost for a single $B S, C_{E P C}$ the core network's capital cost for the deployment of a single eNB, $f_{B W}$ is the backhaul bandwidth (BW) - expressed in $€ / \mathrm{Gbps}-\mathrm{C}_{s t}$ is site costs apart from maintenance cost, backhaul BW for a site's interconnection and $f_{m}$ site maintenance costs. Equations (4) and (5) [1] are the same but Equation (6) is for a small cell. Equations (1)-(3) [18] all have a limitation; imbursement is always made at the end of the investment and this can affect the agreed payment plan or attract a penalty if the MNO defaults on payment. Fluctuation in the interest rate and inflation affect the amount to be paid. The calculation does not take into account the time value for money (TVM), making the model unattractive to investors. Equations (4)-(6) are the TCO for a macrocell and small cell but do not take into consideration whether infrastructure is owned by the MNO or a tower owner. The TCO also does not consider the tower type, which is a principal cost element in tower building, or whether the tower infrastructure is owned by the MNO or rented.

\subsection{Techno-Economic Conventional Model Formulation}

The aim of the study is to develop a risk-free economic model that will mitigate all the limitations of the models used in [5,7]. The model will do away with the effects of TVM, interest rate and inflation control, which are major drawbacks of the models in 1 , 2 and 3 . The model TCO or total cost of investment (TCI) value will be $(1+\alpha)$ times that of the previous model. This makes the developed model more robust and good for MNO, resulting in a good net present value (NPV). The initial capital investment for a $5 \mathrm{G}$ network architecture will be proposed. The initial operational investment for a centralized $5 \mathrm{G}$ network architecture will be proposed.

$$
C_{p}=C_{i}(1+\alpha)\left(\frac{\alpha(1+\alpha)^{\psi}}{(1+\alpha)-1}\right)
$$


Equation (7) is the interest-free, TVM and inflation control model.

5.2. Capex for Telecom 5G Technology Deployment of Modified Picocell (Radio Remote Head, RRH) (gNodeB)

Capex is the sum of money advanced for the implementation of new telecom infrastructure or the purchase of new equipment. Examples are DAS, eNodeB, picocell and EPC. It also comprises the investments made on updating the infrastructure with new hardware, installation, building, back-hauling etc. The costs of installation for the BS nodes alongside the core network, therefore, appertain to this cost category.

Suppose $C_{\text {imvMNO }}$ is the initial Capex, $\alpha$ is the interest for the cost of capital and $C_{c x m c}$ is the repayment amount then, based on Equation (7),

$$
C_{\text {cxpicocell }}=C_{\text {imvpicocell }}(1+\alpha)\left(\frac{\alpha(1+\alpha)^{\psi}}{(1+\alpha)-1}\right) \text {. }
$$

The main advantage of this model over what is being used is that the TCO value will be $(1+M)$ times that of the previous model, making this kind of investment more profitable. The risk level is very low and any increase in the interest rate will have no effect on the return value of the investment.

Assuming that the MNO owns the (mast) telecom tower,

$$
C_{\text {Imvpicocell }}=(M s+M d)\left(C_{C X M C T}+C_{E N B}+C_{E P C}+C_{B H}+C_{o r d}\right) .
$$

For number of gNodeB,

$$
C_{\text {ImvMacro }}=C_{C X M C T}+N p d\left(C_{E N B}+C_{E P C}+C_{B H}+C_{o r d}+C_{b w m}\right)(M s+M d) .
$$

By substituting Equation (10) into (8),

$$
C_{\text {cxpicocell }}=C_{C X M C T}+N\left(C_{E N B}+C_{E P C}+C_{B H}+C_{o r d}+C_{b w m}\right)(M s+M d)(1+\alpha)\left(\frac{\alpha(1+\alpha)^{\psi}}{(1+\alpha)-1}\right) .
$$

\subsection{Opex for Telecom 5G Technology for Modified Picocell (RRH) (gNodeB)}

Opex is the amount of money invested in the cost of the day-to-day operation of the system, depending of the type of operations, cost of rent, vehicle maintenance cost, salaries and wages. $C_{E N B}$ is the cost of the eNodeB, $C_{E P C}$ the cost of the evolved packet, $C_{B H}$ cost of backhauling or transmission networks, $C_{\text {ord }}$ the cost of installation, $M s$ and $M d$ are the number of antennas at the source and destination. If $C_{\text {InvoMNO }}$ is the operation expenditure for the MNO.

$C_{\text {InvoMacro }}=C_{s m}+C_{m e}$, where $C_{s m}$ is the site management cost and $C_{m e}$ is the site material cost.

For $N$ number of sites,

$$
C_{\text {Invopicocell }}=\operatorname{Npd}\left(C_{s m}+C_{m e}\right)(M s+M d) .
$$

For Equation (7),

$$
C_{\text {oxpicocell }}=\frac{C_{\text {Invopicocell }}}{1}(1+\alpha)\left(\frac{\alpha(1+\alpha)^{\psi}}{(1+\alpha)-1}\right) .
$$

By substituting Equation (12) in to (13),

$$
C_{o x M a c r o}=N p d\left(C_{s m}+C_{m e}+C_{\text {Rent }}\right)(M s+M d)\left((1+\alpha)\left(\frac{\alpha(1+\alpha)^{\psi}}{(1+\alpha)-1}\right)\right) .
$$


If the tower is owned by the $\mathrm{MNO} \rightarrow C_{n e t}=0$ and $C_{o x M C}$, the Opex of the telecom (mast) tower will be added to the equation:

$$
C_{\text {oxpicocell }}=C_{o x M C}+N\left(C_{s m}+C_{m e}\right)(M s+M d)\left((1+\alpha)\left(\frac{\alpha(1+\alpha)^{\psi}}{(1+\alpha)-1}\right)\right) .
$$

\subsection{Total Cost of Investments for Modified Picocell (RRH) (gNodeB)}

TCO or TCI is a single value that represents the lifespan of a capital purchase. It is a financial estimate that helps in determining the direct and indirect costs of a product or services. It is the sum of the operational cost and the capital cost. It helps in making critical agreement vs. buy comparisons. It concerns vendor selection, prioritization of capital acquisition, and overall corporate budgeting.

$$
\begin{gathered}
T C I_{\text {Picocell }}=C_{c x M C}+C_{o x \text { Macro }} \\
T C I_{\text {Picocell }}=C_{c x M C T}+N p d\left(C_{E N B}+C_{E P C}+C_{B H}+C_{o r d}\right)(M s+M d)\left((1+\alpha)\left(\frac{\alpha(1+\alpha)^{\psi}}{(1+\alpha)-1}\right)\right) \\
+C_{o x M C}+N p d\left(C_{s m}+C_{m e}\right)(M s+M d)\left((1+\alpha)\left(\frac{\alpha(1+\alpha)^{\psi}}{(1+\alpha)-1}\right)\right)
\end{gathered}
$$

factorization of Equation (17):

$$
T C I_{\text {Picocell }}=C_{c x M C T}+C_{o x M C T}\left((1+\alpha)\left(\frac{\alpha(1+\alpha)^{\psi}}{(1+\alpha)-1}\right)\right) \times N p d\left(\begin{array}{c}
C_{E N B}+C_{E P C}+C_{B H} \\
+C_{o r d}+C_{b w m}+C_{s m}+C_{m e}
\end{array}\right)(M s+M d)
$$

If the MNO is renting the tower, then $C_{c x M C T}, C_{o x M C T}$ and equal to zero (0) and $C_{\text {Rent }}$ will be added to the Opex of the MNO. Therefore, Equation (18) will become:

$$
T C I_{\text {Picocell }}=(1+\alpha)\left(\frac{\alpha(1+\alpha)^{\psi}}{(1+\alpha)-1}\right)(M s+M d) N\left(\begin{array}{l}
C_{E N B}+C_{E P C}+C_{B H}+C_{o r d}+C_{b w m} \\
+C_{s m}+C_{m e}+C_{\text {Rent }}+C_{b w o m}
\end{array}\right)
$$

5.5. Capex for Telecom 5G Technology Deployment with Modified Distributed Antenna System (MDAS) (RHH)

Capex includes the cost of purchasing new infrastructure and integrating new items when existing equipment is updated. In MDAS (RHH) BS costs are given by the following equation: $\left(C_{E N B}+C_{E P C}+C_{B H}+C_{\text {ord }}\right)$. If there are $N p d$ BSs, then $N p d\left(C_{E N B}+C_{E P C}+\right.$ $\left.C_{B H}+C_{o r d}\right)$ is the cost for all the BS. The total capital cost for a year will then be given by

$$
C_{c x D A S R R H}=(1+\alpha)\left(\frac{\alpha(1+\alpha)^{\psi}}{(1+\alpha)-1}\right) N\left(C_{E N B}+C_{E P C}+C_{B H}+C_{o r d}\right)
$$

5.6. Opex for Telecom 5G Technology for MDAS (RRH)

The Opex for a picocell (small cell) will be the cost of operation and maintenance of the network and site rental, if $C_{s m}$ and $C_{r}$ is the site rental cost then for $N \mathrm{BS}$, the sum of the Opex variable will be $N\left(C_{s m}+C_{r}\right)$.

$$
C_{\text {oxMDASRRH }}=(1+\alpha)\left(\frac{\alpha(1+\alpha)^{\psi}}{(1+\alpha)-1}\right) N\left(C_{s m}+C_{r}\right)
$$

The TCO or cost of investment for the MDAS(RRH) is the sum of the total Capex and the total Opex.

$$
\begin{gathered}
T C I_{M D A S R R H}=C_{c x R R H}+C_{o x R R H} \\
T C I_{M D A S R R H}=N(1+\alpha)\left(\frac{\alpha(1+\alpha)^{\psi}}{(1+\alpha)-1}\right)\left(C_{E N B}+C_{B H}+C_{o r d}+C_{s m}+C_{r}\right)
\end{gathered}
$$




\subsection{Distributed Indoor Solution with Femtocell}

LTE femtocell technology is used in the indoor environment. The introduction of femtocell brings about a reduction in the deployment of macrocell sites and reduces the millions of dollars operators spend on building macrocell sites. Femtocells provide exceptional improved coverage and capacity, resulting in revenue generation from services such as internet service, video and data service. When femtocell technology is used, the electricity bill is no longer the responsibility of the operators. The ability of a femtocell to do automatic configuration, self-organization, synchronization and timing eliminates the cost of operation and maintenance. Compared to macrocell, microcell and picocell, the deployment of femtocell will reduce capital and operating expenditure, thus reducing the TCI or TCO to the MNO, as shown in Equation (24).

$$
T C I_{\text {DFemto }}=N f d(1+\alpha)\left(\frac{\alpha(1+\alpha)^{\psi}}{(1+\alpha)-1}\right)\left(C_{E N B}+C_{B H}+C_{E P C}\right)
$$

\subsection{Total Cost of Investment or Cost of Ownership for Distributed Wireless Network}

The total cost of investment for the DWNA designed with picocell and DAS is defined as the sum of all the TCI for the number of BSs deployed.

$$
\begin{array}{r}
T C I_{D W N}=T C I_{\text {Picocell }}+T C I_{D A R R H}+T C I_{\text {DFemto }} \\
T C I_{D W N}=(1+\alpha)\left(\frac{\alpha(1+\alpha)^{\psi}}{(1+\alpha)-1}\right)(M s+M d) N\left(\begin{array}{c}
C_{E N B}+C_{E P C}+C_{B H}+C_{\text {ord }} \\
+C_{s m}+C_{m e}+C_{\text {Rent }}
\end{array}\right)+ \\
N d d(1+\alpha)\left(\frac{\alpha(1+\alpha)^{\psi}}{(1+\alpha)-1}\right)\left(C_{E N B}+C_{B H}+C_{E P C}+C_{o r d}+C_{s m}+C_{r}\right)+ \\
N f d(1+\alpha)\left(\begin{array}{l}
\alpha(1+\alpha)^{\psi} \\
(1+\alpha)-1
\end{array}\right)\left(C_{E N B}+C_{B H}+C_{E P C}\right) \\
T C I_{D W N}=(1+\alpha)\left(\frac{\alpha(1+\alpha)^{\psi}}{(1+\alpha)-1}\right)(M s+M d) N p\left\{\begin{array}{l}
\left(\begin{array}{l}
C_{E N B}+C_{E P C}+C_{B H}+C_{o r d} \\
+C_{s m}+C_{m e}+C_{\text {Rent }} \\
N d\left(C_{E N B}+C_{B H}+C_{E P C}+C_{o r d}+C_{s m}+C_{r}\right)+ \\
N f\left(C_{E N B}+C_{B H}+C_{E P C}\right)
\end{array}\right)
\end{array}\right.
\end{array}
$$

\section{Network Capacity and Throughput for Distributed Wireless Network}

Capacity planning in a telecommunications network is studied through the novel application of inventory control techniques aimed at meeting the demand for a certain service level. In transmission, we define capacity as the maximum transmission rate of the link, whether microwave, radio or fiber. Capacity significantly affects the communication quality of $3 \mathrm{G}$ and $4 \mathrm{G}$ in terms of maximum achievable throughput and end-user's satisfaction and 5G LTE technology is to address this challenge. Capacity evaluation is, therefore, of great importance for both $5 \mathrm{G}$ network planning and management. According to Shannon's capacity theory, the total system capacity in a 5G wireless cellular network is the sum of the capacity of all sub-channels deployed in heterogeneous networks. In 5G technology, the total network capacity can be increased by increasing the network coverage through heterogamous network implementation of picocells, small cells (DAS), mobile femtocells and D2D clustering and by increasing the number of sub-channels, cognitive radio networks, massive MIMO, mmWaves and visible light communications. Cloud or centralized regional area networks dynamically allocate on demand using the coordination of multiple antenna ports or cells and joint processing of radio signals and centralizes various radio resources to manage.

In this section we drive the capacity model for a DWNA by applying Shannon capacity theory realized in [19] and the model for the throughput per user will be presented. 
If the transmission bandwidth is given by $B$, and the signal-to-noise interference ratio [20] is given as $\beta$, then applying Shannon capacity [21] on macrocell, picocell, small cell and femtocell results in:

$$
\begin{gathered}
D_{c P i c}=B \log _{2}\left(1+\beta_{\text {Pic }}\right) \\
D_{c P i c}=N B \log _{2}\left(1+\beta_{\text {Pic }}\right) \\
D_{c s}=N_{s} B_{s} \log _{2}\left(1+\beta_{s c}\right) \\
D_{c p}=N_{p} B_{p} \log _{2}\left(1+\beta_{p}\right) \\
D_{c f}=N_{f} B_{f} \log _{2}\left(1+\beta_{f}\right) \\
D C_{D w N A}=\sum H E T-N E T \sum \operatorname{channel}\left[B w \log _{2}(1+\beta)\right] \\
\text { Thru per User }=\frac{D C_{D w N A}}{P_{T}}
\end{gathered}
$$

\section{Model Verification}

The 5G technology will require numerous NR to be deployed to achieve dense coverage, high capacity, high throughput and ultra-reliable low latency communication. 5G will require shorter inter-site distances (ISD), meaning $\mathrm{MNO}$ will deploy many more sites than in $4 \mathrm{G}$. Both old $4 \mathrm{G}$ and $3 \mathrm{G}$ of $1 \mathrm{GHz}$ to $2.6 \mathrm{GHz}$ will be required for high coverage, as well as a new high-band spectrum between $3.5 \mathrm{GHz}$ and $40 \mathrm{GHz}$ for high capacity. The deployment will require the installation of RRH (4T4R, 8T8R, 16T16R, 16T16R, 32T32R), with massive MIMO antenna and fronthaul (fiber optics) on the tower (see the centralized architecture in Figure 1). Based on the needs of 5G technology, the cheaper and easier way of deploying this in Ghana and the rest of Africa would be the implementation of centralized architecture in collaboration with network sharing to enable MNO to meet the ISD of $5 \mathrm{G}$ technology. This section will report on the testing of the model using the Mobile telecommunication networks (MTN) network in the Tema metropolitan area. Table 1 is the TCO cost parameter and independent variables which will be used for the model verification. Tema has an area of $565 \mathrm{~km}^{2}$ [22] and a population of about 292,700 living

\begin{tabular}{|c|c|c|c|}
\hline Parameter & Description & Value & Range of Analysis \\
\hline \multicolumn{4}{|c|}{ Picocell } \\
\hline$C_{e N B}$ or $g N B$ & Capital cost of eNB or gNB & $\$ 1250.00$ & {$[\$ 400,1600]$} \\
\hline Crrh & Cost of RRH & 299 & \\
\hline$C_{E P C}$ & Cost of core for deploying single gNB & $\$ 137.50$ & {$[\$ 50,175]$} \\
\hline$N p d$ & Number of gNB or NR deployed & 1 & {$[1,99]$} \\
\hline$n$ & Period of repayment plan of a site in years & 6.00 & {$[2,20]$} \\
\hline$m$ & Annual interest rate & 0.08 & {$[2.5,12.5]$} \\
\hline$C_{s m}$ & Cost of site maintenance/year & $\$ 3800.00$ & {$[\$ 1150,5550]$} \\
\hline$C_{\text {oxmct }}$ & $\begin{array}{l}\text { Cost of running the operation (passive, active } \\
\text { and others) }\end{array}$ & $\$ 1338.75$ & {$[\$ 446.25,1338.75]$} \\
\hline$C_{c x m c t}$ & $\begin{array}{c}\text { Cost of investment of the passive } \\
\text { infrastructure/year }\end{array}$ & $\$ 17,727.95$ & \\
\hline$C_{B H m}$ & Annual cost of front and backhaul & $\$ 6000.00$ & {$[\$ 2400,7200]$} \\
\hline bwm & Cost of site interconnection & 10 & {$[5,15]$} \\
\hline
\end{tabular}
in 40,956 houses. We will verify the economic performance of the models for MPICOC (small cell), MDAS (RRH) and femtocell [18]. An SA is performed on MPICOC (small cell), MDAS (RRH) and femtocell in a $50 \mathrm{~km}^{2}$ area. Finally, a sensitivity analysis [23] is used to assess the influence of a key variable on the Capex, Opex and TCO of the MPICOC (small cell), MDAS (RRH, and femtocell.

Table 1. Total cost of ownership (TCO) cost parameter and indispensable variables. 
Table 1. Cont.

\begin{tabular}{|c|c|c|c|}
\hline Parameter & Description & Value & Range of Analysis \\
\hline$C_{m e}$ & Cost of installation material & $\$ 980$ & \\
\hline$C_{\text {ord }}$ & Cost of installation & $\$ 650$ & \\
\hline Lbw & $\begin{array}{l}\text { Backhauling cost per site annually for MPICOC } \\
\text { BW } \$ \text { Gbps }\end{array}$ & 1300 & {$[\$ 585,1755]$} \\
\hline Bs & MIMO antennas at the source of MPICOC & 128 & {$[2,4,8,16,64,128,256,512]$} \\
\hline Ue & $\begin{array}{c}\text { MIMO antennas at the destination of user } \\
\text { equipment }\end{array}$ & 8 & {$[2,4,8,16,64,128,256,512]$} \\
\hline Parameter & Modified DAS (RRH) & Value & Range of Analysis \\
\hline$C_{e n b p}$ & Capital cost for a single small cell (MDAS) & $\$ 850.00$ & {$[\$ 5950,17,850]$} \\
\hline $\mathrm{RRH}$ & Cost of $(\mathrm{RRH})$ & $\$ 78.00$ & \\
\hline$C_{\text {epcp }}$ & Cost of core for deploying single gNB & $\$ 80.00$ & \\
\hline$N d p$ & Number of MDAS eNB & 130 & {$[10,130]$} \\
\hline$C_{s p}$ & Cost of site maintenance & $\$ 270.00$ & {$[\$ 78.84,236.54]$} \\
\hline$B s p$ & MIMO antennas at the source of MDAS & 64 & {$[2,4,8,16,64,128,256,512]$} \\
\hline Uep & $\begin{array}{l}\text { MIMO antennas at the destination of user } \\
\text { equipment }\end{array}$ & 4 & {$[2,4,8,16,64,128,256,512]$} \\
\hline $\mathrm{C}_{\mathrm{BHp}}$ & Fronthaul cost for optical fiber & $\$ 6900.00$ & \\
\hline Cbhp & $\begin{array}{l}\text { Backhauling cost per site annually for MDAS } \\
\text { BW } \$ / \text { Gbps }\end{array}$ & $\$ 5$ & \\
\hline Csit & Cost of site interconnection & 10 & {$[5,15]$} \\
\hline Cbhp Csit & Backhaul costs with provided BW\$ & 50 & \\
\hline Parameter & Femtocell & Value & Range of Analysis \\
\hline$C_{e n b f}$ & Capital cost for a single Bs & $\$ 110.00$ & \\
\hline$B h f$ & $\begin{array}{l}\text { Backhauling cost per site annually for femtocell } \\
\text { BW } \$ / G b p s\end{array}$ & $\$ 544.00$ & \\
\hline$N f$ & Number of femtocells and EPCs needed & 120 & {$[1,120]$} \\
\hline$C_{E P C f}$ & $\begin{array}{l}\text { Capital cost for the deployment of a single eNB } \\
\text { on the core network }\end{array}$ & $\$ 80.00$ & \\
\hline$B s f$ & MIMO antennas at the femtocell source & 128 & {$[2,4,8,16,64,128,256,512]$} \\
\hline Uef & $\begin{array}{l}\text { MIMO antennas at the destination user } \\
\text { equipment }\end{array}$ & 8 & {$[2,4,8,16,64,128,256,512]$} \\
\hline$B h f c$ & Backhauling cost & $\$ 38.50$ & \\
\hline
\end{tabular}

\section{Simulation Result for Modified Picocell and MDAS}

Table 2 indicates the simulation result of the model in Equation (19) when the MNO owns the tower infrastructure. At 7.5\% interest rate, SA shows that the Capex, Opex and TCO are directly proportional to the number of cells deployed. Table 3 shows the SA when the MNO shares the tower infrastructure and will only pay rent to the tower company. The result indicates an average saving in the TCO of $15.34 \%$ when the MNO shares tower infrastructure. Table 4 indicates the SA result for MDAS when the tower infrastructure is owned by the MNO and Table 5 shows when the MNO is leasing the tower infrastructure from the tower company, resulting in $10.6 \%$ savings in TCO. More significantly, MNOs must practice tower infrastructure sharing when deploying 5G technology, more traffic offload should occur, and MDAS must be used to reduce the cost of Capex, Opex and TCO. 
Table 2. Modified picocell, 7.5\%, owned tower.

\begin{tabular}{cccc}
\hline Sites & Capex k\$ & Opex k\$ & TCO k\$ \\
\hline 1 & 286.0 & 80.0 & 366.1 \\
2 & 572.0 & 160.1 & 732.1 \\
3 & 858.1 & 240.1 & 1098.2 \\
4 & 1144.1 & 320.1 & 1464.2 \\
5 & 1430.1 & 400.1 & 1830.3 \\
\hline
\end{tabular}

Table 3. Modified picocell, $7.5 \%$, shared tower.

\begin{tabular}{cccc}
\hline Sites & Capex k\$ & Opex k\$ & TCO k\$ \\
\hline 1 & 268.3 & 44.5 & 312.8 \\
2 & 536.6 & 89.0 & 625.6 \\
3 & 804.9 & 133.6 & 938.4 \\
4 & 1073.2 & 167.9 & 1241.1 \\
5 & 1341.5 & 196.7 & 1538.2 \\
\hline
\end{tabular}

Table 4. Modified DAS, 7.5\%, owned tower.

\begin{tabular}{cccc}
\hline Sites & Capex k\$ & Opex k\$ & TCO k\$ \\
\hline 1 & 129.0 & 4.2 & 133.2 \\
2 & 258.1 & 8.4 & 266.5 \\
3 & 387.1 & 12.6 & 399.7 \\
4 & 516.1 & 16.8 & 532.9 \\
5 & 645.1 & 21.0 & 666.2 \\
\hline
\end{tabular}

Table 5. Modified DAS, 7.5\%, shared tower.

\begin{tabular}{cccc}
\hline Sites & Capex k\$ & Opex k\$ & TCO k\$ \\
\hline 1 & 114.9 & 4.2 & 119.1 \\
2 & 229.8 & 8.4 & 238.2 \\
3 & 344.7 & 12.6 & 357.3 \\
4 & 459.6 & 16.8 & 476.4 \\
5 & 574.5 & 21.0 & 595.5 \\
\hline
\end{tabular}

\subsection{Sensitivity Analysis}

Key Findings of Sensitivity Analysis and Future Work

Figure 2 shows the SA of an MPICOC and an MDAS system, where the MNO owns the tower infrastructure. When comparing the Capex, Opex and TCO, it is evident that the DAS yields a significant reduction in cost. The Capex of the picocell is $54.2 \%$ higher than that of the MDAS; the Opex is $98 \%$ higher and the TCO for MPICOC $63.8 \%$ higher than that of the MDAS. In this architecture all the traffic of the MDAS is routed to the MPICOC through a fronthaul fiber to the RRH of the DAS. The energy consumption of the DAS is $83 \%$ less than that of the picocell. Figure 3 shows the SA of the MPICOC and MDAS, where the $\mathrm{MNO}$ is sharing the tower infrastructure. When the MNO only pays rent to the tower company, the result is a $22 \%$ reduction in Capex, but the operational cost is constant. Figure 4 shows the effect of deploying MIMO on TCO for MDAS. The graphs show that the activation of MIMO will increase the capital and operational cost and the TCO. The analysis shows that massive MIMO technology is cheaper when the number of antennas activated are low; it is not economically viable when the number of antennas exceeds 500. In Figure 5, which reflects the analysis of operational cost between the MPICOC and MDAS, the SA shows that the operational cost of the MPICOC is $69.6 \%$ higher than that of MDAS. Opex will increase for both technologies when the number of antennas rises to above 12. MNOs must take note of these essential parameters when implementing 5G. It is more economical 
to share tower infrastructure than to own it. When implementing MIMO technology, MNO must not operate more than 500 massive MIMO and also when deploying a distribution system they must use more DAS in the architecture so they can offload more traffic to the picocell or any major cell sites. Future work could involve consideration of a new radio with distributed core wireless network architecture and a technoeconomic analysis comparison of the TCO of the distributed core network architecture.

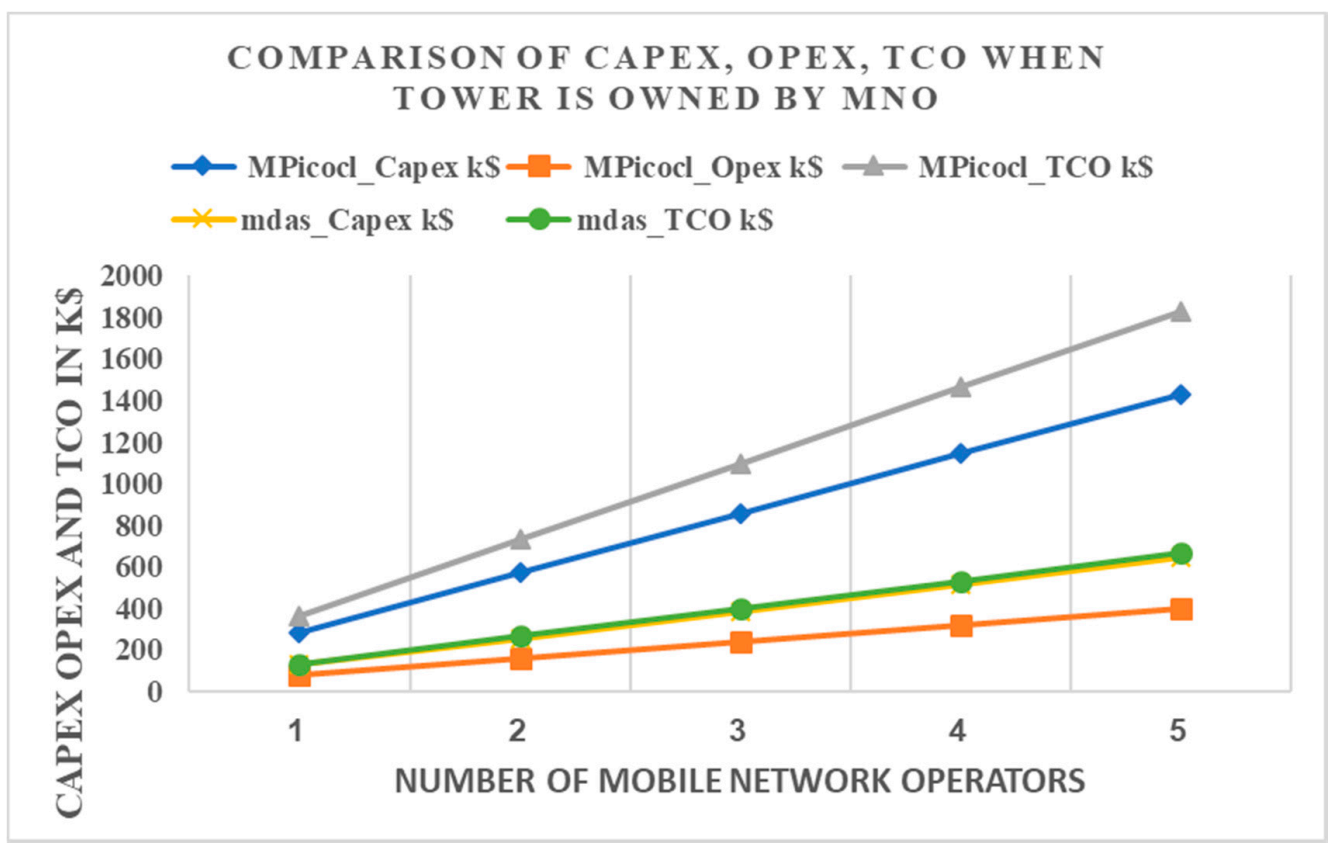

Figure 2. Sensitivity analysis of Capex, Opex and TCO for MPICOC and MDAS when mobile network operator (MNO) owns the tower.

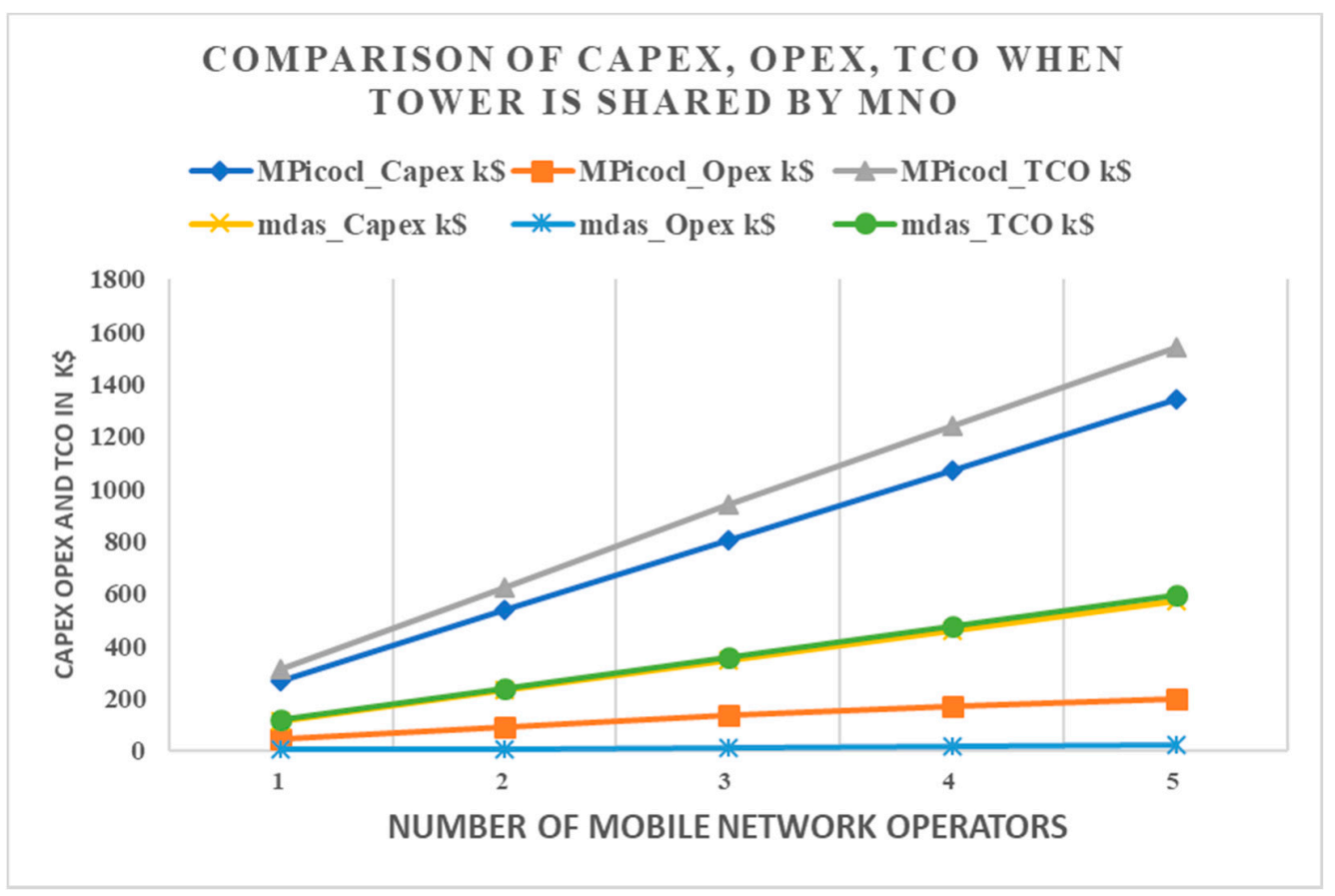

Figure 3. Sensitivity analysis of Capex, Opex and TCO for MPICOC and MDAS when MNO shares the tower. 


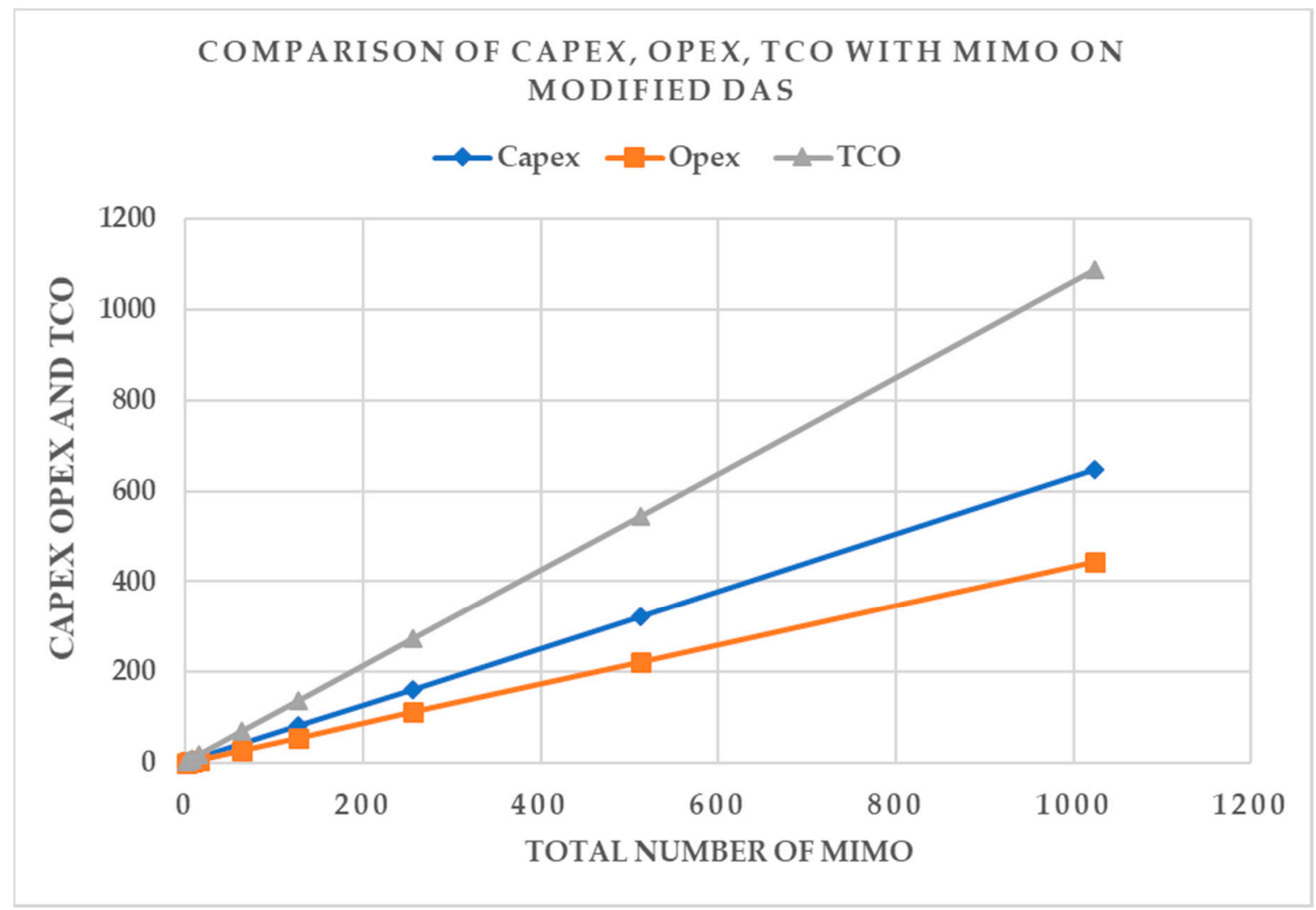

Figure 4. Sensitivity analysis for MDAS and MIMO.

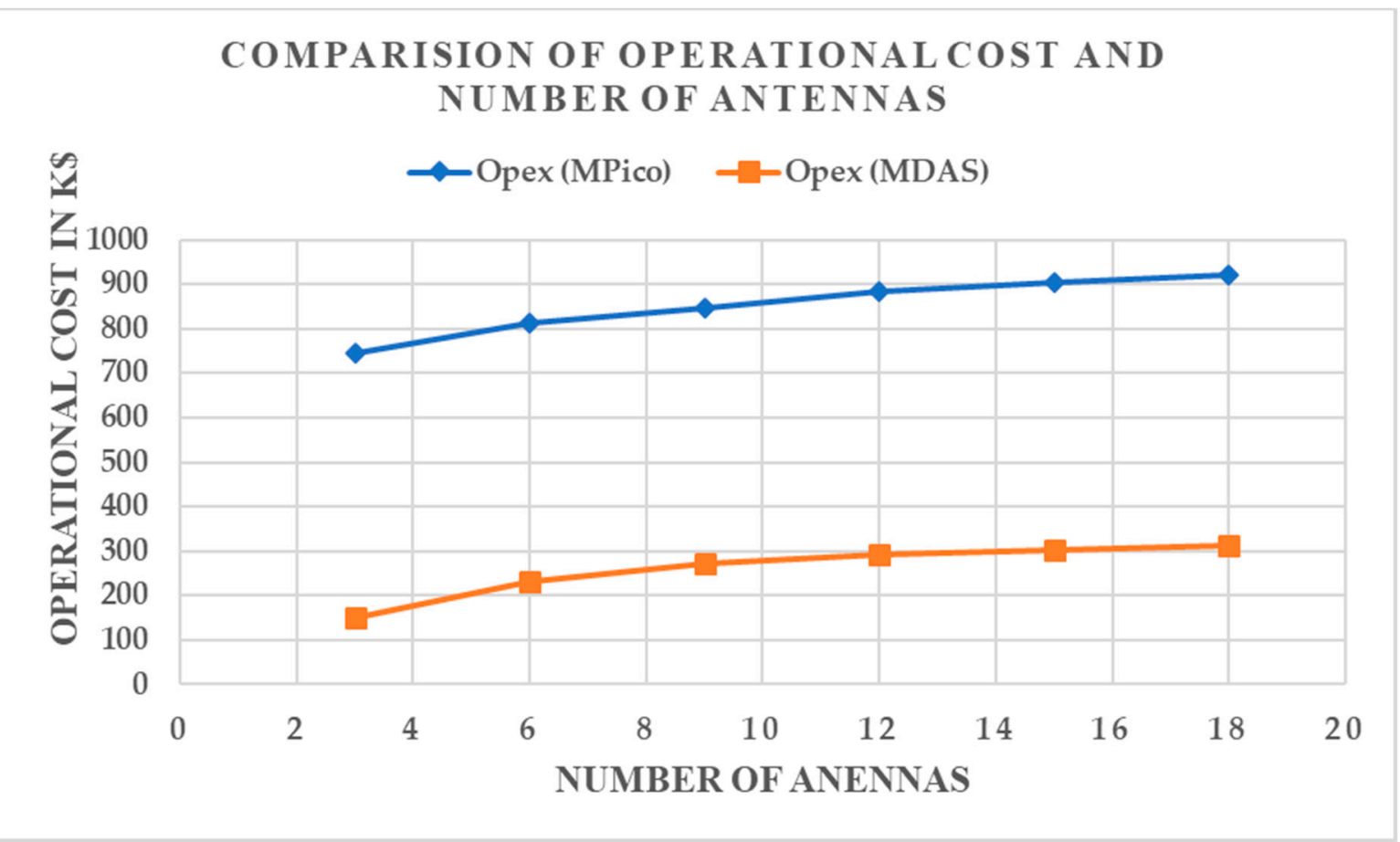

Figure 5. Sensitivity analysis on Opex for MPICOC and MDAS with the number of antennas. 


\section{Conclusions}

Fifth-generation technology will require a complete overhaul of the telecommunication architecture design and implementation. In this paper, we present distributed network architecture for the next generation (5G) technology using a proposed MPICOC and MDAS, which will provide dense coverage, high capacity and low latency. An economical model for Capex, Opex and TCO for MPICOC, MDAS and distributed architecture was also presented. A mathematical model was presented for capacity and throughput for a distributed wireless network. A model was simulated for the MPICOC and MDAS when the MNO owns the tower infrastructure and when the tower infrastructure belongs to tower companies, using numerical data from the national communication system of Ghana and MNO. We also performed SA to evaluate the Capex, Opex and TCO with MIMO on MPICOC and MDAS. SA was performed to evaluate the Capex, Opex, and TCO with MIMO on MPICOC and MDAS. The result shows that MNO must take note of these essential parameters when implementing 5G.

Author Contributions: Conceptualization, I.A.G.; methodology, I.A.G.; Software, I.A.G.; formal analysis, I.A.G.; investigation, I.A.G.; Supervision, N.I.N.; writing-review and editing, I.A.G. and N.I.N.; visualization, I.A.G. and N.I.N.; writing original-original draft preparation, I.A.G. All authors have read and agreed to the published version of the manuscript.

Funding: This study received no external funding.

Data Availability Statement: No applicable.

Conflicts of Interest: Author declares no conflict of interest.

\section{References}

1. Bouras, C.; Kollia, A.; Papazois, A. Dense Deployments and DAS in 5G: A Techno-Economic Comparison. Wirel. Pers. Commun. 2017, 94, 1777-1797. [CrossRef]

2. Bouras, C.; Kokkinos, V.; Kollia, A.; Papazois, A. Analyzing-Small-Cells-and-Distributed-Antenna-Systems-from-TechnoEconomic-Perspective.pdf. Int. J. Wirel. Netw. Broadband Technol. 2017, 6, 45-64. [CrossRef]

3. Teterin, V.; Hurley, S. Optimized network dimensioning and planning for WiMAX technology. Ad Hoc Netw. 2014, 13, 381-403. [CrossRef]

4. Seremet, I.; Čaušević, S. Evolving IP/MPLS Network in Order to Meet 5G Requirements. March 2019, 4, 13-18.

5. Krizanovic, V.; Zagar, D.; Grgic, K. Techno-Economic Analyses of Wireline and Wireless Broadband Access Networks Deployment in Croatian Rural Areas. In Proceedings of the 11th International Conference on Telecommunications, Graz, Austria, 15-17 June 2011; pp. 265-272.

6. Smail, G.; Weijia, J. Techno-economic analysis and prediction for the deployment of 5G mobile network. In Proceedings of the 2017 20th Conference on Innovations in Clouds, Internet and Networks (ICIN), Paris, France, 7-9 March 2017; pp. 9-16.

7. Bouras, C.; Kokkalis, S.; Kollia, A.; Papazois, A. Techno-economic analysis of MIMO das in 5G. In Proceedings of the 2018 11th IFIP Wireless and Mobile Networking Conference (WMNC), Prague, Czech Republic, 3-5 September 2018.

8. Bouras, C.; Kokkalis, S.; Kollia, A.; Papazois, A. Techno-economic comparison of MIMO and DAS cost models in 5G networks. Wirel. Netw. 2018, 6, 1-15. [CrossRef]

9. Verbrugge, S.; Pasqualini, S.; Westphal, F.J.; Jäger, M.; Iselt, A.; Kirstädter, A.; Chahine, R.; Colle, D.; Pickavet, M.; Demeester, P. Modeling operational expenditures for telecom operators. In Proceedings of the 2005 Conference on Optical Network Design and Modeling Towar. Broadband-for-All Era, ONDM 2005, Milan, Italy, 7-9 February 2005; pp. 455-466.

10. Antonopoulos, A. Bankruptcy Problem in Network Sharing: Fundamentals, Applications and Challenges. IEEE Wirel. Commun. 2020, 27, 81-87. [CrossRef]

11. Eal, D.E.M.; Boiardi, S. Radio Planning and Management of Energy-Efficient Wireless. Ph.D. Thesis, École Polytechnique de Montréal, Montreal, QC, Canada, 2014.

12. Oughton, E.J.; Frias, Z. The cost, coverage and rollout implications of 5G infrastructure in Britain. Telecomm. Policy 2018, 42, 636-652. [CrossRef]

13. McKinsey \& Company. The Road to 5G: The Inevitable Growth of Infrastructure Cost I McKinsey; McKinsey Co.: New York, NY, USA, 2018; pp. 1-8.

14. Habibi, M.A.; Nasimi, M.; Han, B.; Schotten, H.D. A Comprehensive Survey of RAN Architectures toward 5G Mobile Communication System. IEEE Access 2019, 7, 70371-70421. [CrossRef]

15. Ruggeri, E.; Tsakyridis, A.; Vagionas, C.; Leiba, Y.; Kalfas, G.; Pleros, N.; Miliou, A. Multi-User V-Band Uplink Using a Massive MIMO Antenna and a Fiber-Wireless IFoF Fronthaul for 5G mmWave Small-Cells. J. Light. Technol. 2020, 38, 5368-5374. [CrossRef] 
16. Kalfas, G.; Vagionas, C.; Antonopoulos, A.; Kartsakli, E.; Mesodiakaki, A.; Papaioannou, S.; Maniotis, P.; Vardakas, J.S.; Verikoukis, C.; Pleros, N. Next Generation Fiber-Wireless Fronthaul for 5G mmWave Networks. IEEE Commun. Mag. 2019, 57, 138-144. [CrossRef]

17. Bouras, C.; Kokkinos, V.; Papazois, A. Financing and Pricing Small Cells in Next-Generation Mobile Networks. In International Conference on Wired/Wireless Internet Communications; Springer: Cham, Switzerland, 2014; pp. 41-54.

18. Yunas, S.F.; Ansari, W.H.; Valkama, M. Technoeconomical Analysis of Macrocell and Femtocell Based HetNet under Different Deployment Constraints. Mob. Inf. Syst. 2016, 2016, 6927678. [CrossRef]

19. Chen, L. Coverage Planning and Resource Allocation in Broadband Cellular Access-Optimization Models and Algorithms. Ph.D. Thesis, Linköping University, Linköping, Sweden, 2010.

20. Kayastha, N.; Niyato, D. A Review of Radio Resource Management in FemtoCell from Interference Control Perspective. ECTI Trans. Comput. Inf. Technol. (ECTI-CIT) 2017, 11, 103-128. [CrossRef]

21. Chandrasekhar, V.; Andrews, J.G. Uplink capacity and interference avoidance for two-tier femtocell networks. IEEE Trans. Wirel. Commun. 2009, 8, 3498-3509. [CrossRef]

22. Norty, E. National Analytical Report 2010 Population and Housing Censes; Ghana Statistical Service: Accra, Ghana, 2013.

23. Thabane, L.; Mbuagbaw, L.; Zhang, S.; Samaan, Z.; Marcucci, M.; Ye, C.; Thabane, M.; Giangregorio, L.; Dennis, B.; Kosa, D.; et al. A tutorial on sensitivity analyses in clinical trials: The what, why, when and how. BMC Med. Res. Methodol. 2013, 13, 1-12. [CrossRef] [PubMed] 